\title{
Review of: "Aerial transmission of the SARS-CoV-2 virus through environmental e-cigarette aerosol: implications for public policies"
}

\author{
Andrés Lanzós
}

Potential competing interests: The author(s) declared that no potential competing interests exist.

I must disclaim that I did not read fully the current manuscript. And that is because I found several invalidating points (mentioned below) that discouraged me to continue. In summary, I highly doubt the correctness of the current manuscript. I even have some doubts regarding the honesty of authors. However, all can be due to honest errors and my misunderstanding. Therefore, I would suggest authors to take a look at my suggestions below and improve the quality and language of the manuscript. Specially, how previous work is handled and cited.

1. Direct source should be cited for [10], not simply a news article.

2. "nearly 2.7 billion jobs were lost " is claimed by authors. Checking source [11], that number refers to jobs in regions with partial or total lockdown. So such claim is not correct, please amend it. In fact, loosing $87 \%$ of world's jobs seems quite insane, almost apocalyptic.

3. I could not find any information regarding the following claim in the cited source [13]: Forced isolation has contributed to anxiety, depression and a rise in the intake of psychoactive stimulants, alcohol, cigarettes and nicotine products in certain individuals to alleviate tension and negative feelings [13]

4. The entire MedRxix manuscript that is referred to in this text [18] (from same authors), is based on inferential science and not empirical evidence (to my understanding). Therefore, to me, this manuscript fails "to address the lack of prior research and evidence", as authors claim. In fact, these two manuscripts fall in the same criteria that authors have described here for other manuscripts: "However, there is no empiric evidence (nor previous proper research ${ }^{\star \star}$ ) of aerial transmission of the SARS-CoV-2 virus (or any pathogen) through environmental e-cigarette aerosol (ECA) or environmental tobacco smoke (ETS) exhaled by infected vapers or smokers."

5. Following that claim that "there is no empiric evidence", empiric evidence indeed exist. A quick search reveals at least three papers with (kind of) empirical evidence in this regard (one of them heavily criticized by some researchers):

https://www.sciencedirect.com/science/article/pii/S1054139X20303992 
https://www.sciencedirect.com/science/article/pii/S2211335520302126

https://respiratory-research.biomedcentral.com/articles/10.1186/s12931-020-01396-y

And authors actually mention it in the current manuscript. So, authors seem incoherent by refusing their own claim in the same manuscript. I would suggest improving the coherence of the text.

6. Authors refer to the previous papers like this "(see [36] for a review of these studies and [37] for critical appraisal)". Checking source [36] and [37], I found that they are completely unrelated manuscripts from 2019 (even before Covid19 was detected), and one of them comes from same author (so self-citation). Therefore, that sentence has no sense at all. 